\title{
Distortions and fluctuations in topographic memory
}

\author{
MARIE-DOMINIQUE GIRAUDO and JEAN PAILHOUS \\ Université d'Aix-Marseille II, Marseille, France
}

\begin{abstract}
Two experiments dealing with the learning of a space by map or by navigation approached the questions of equivalency of the cognitive processes involved in spatial information and of response fluctuation. In the first experiment, 11 subjects were asked to situate, six times, 18 locations on a blank map. In the second experiment, the subjects were first given 3 min to learn a map with 12 locations marked, and then asked to reproduce it. The task was repeated six times, using three different maps. This gave us several trials per subject, so that distortion could be distinguished from response fluctuation. In Experiment 1, the range of values was the same for response inaccuracy and response fluctuation; in Experiment 2, the range was greater for response inaccuracy than for response fluctuation. The results showed that space learning by navigation and space learning by map involve different cognitive processes.
\end{abstract}

The current research addresses an old debate about the memory coding of spatial information of well-known spaces. Some authors assume that the mental representation preserves Euclidean properties such as angle and distance relationships (Golledge \& Spector, 1978; Kosslyn \& Pomerantz, 1977). Others assume that the cognitive map represents a Minkowskian metric (Richardson, 1981) or a topological coding (Golledge \& Hubert, 1982; Tobler, 1976). The latter approaches presumably lead to varying degrees of distortion relative to real space, that is, relative to a cartographic representation, the most convenient method (Golledge, Smith, Pellegrino, Doherty, \& Marshall, 1985).

\section{Distortion}

The debate about the nature of spatial coding in memory has been propagated by numerous empirical results that have shown, on the one hand, considerable accuracy in subjects' spatial estimates (Baird, Merrill, \& Tannenbaum, 1979; Gärling, Böök, \& Ergezen, 1982; Kosslyn, Pick, \& Fariello, 1974) and, on the other hand, inconsistencies in spatial estimates (Moar \& Bower, 1983) and the use of heuristics to simplify coding (Byrne, 1979). Numerous factors likely to explain these contradictory results have been identified. Some such factors are related to individual characteristics. For example, Kozlowski and Bryant (1977) found substantial interindividual differences in distance and location estimates linked to spatial orientation skills. Golledge, Briggs, and Demko (1969) showed

This work was supported by the Centre National de la Recherche Scientifique. The authors wish to thank Vivian Waltz for her assistance with the English version of this article. The authors' mailing address is $\mathrm{Cog}$ nition \& Mouvement, URA CNRS 1166, Université d'Aix-Marseille II, Faculté de Médecine, 1BHOP, Traverse Charles Susini, 13388 Marseille Cedex 13, France.

-Accepted by previous editor, Margaret Jean Intons-Peterson that environmental familiarity was important for distance estimates. Gärling, Böök, Lindberg, and Nilsson (1981) also found important interindividual differences which they ascribed either to the mode of transportation used or to the subject's motivation. Baird, Wagner, and Noma (1982) identified factors linked to the characteristics of the task, and found differences related to the type of estimate made (pairwise judgment or direct mapping). Another problem is that there are important differences in the scale of the spaces investigated. For instance, Kosslyn et al.'s (1974) experiment was performed in a $25-\mathrm{m}^{2}$ room, whereas Baird et al.'s (1979) was conducted in a 800-m-sided campus. Byrne (1979) and Moar and Bower (1983) ran their experiments in a city (significantly increasing scale and complexity). Finally, these various explanations emphasized the different types of spatial knowledge and the different procedures available to make spatial judgments. However, these studies did not investigate the question of the conservation of Euclidean properties of real spaces.

A procedure that allows us to systematically compare a space learned by navigating within it and a space learned by perusing a map may approach this question. Indeed, learning a space by reading a map guarantees that certain types of coding will be used, because subjects directly memorize the spatial relationships between objects. If strong familiarity with a space, acquired by intense navigation within it, allows subjects to develop configurational knowledge with Euclidean properties (i.e., a maplike representation of object locations), then the resulting cognitive map should be equivalent to the one constructed through direct perception of a map. In this case, there is no reason for the processes used by the subjects to produce estimates to be different.

Thorndyke and Hayes-Roth (1982) compared memorized images of spaces acquired from map learning with those acquired from navigation. Using distance estimates, direction estimates, and location estimates, the authors 
showed that map learning was superior. They ascribed this superiority to the relative ease with which global relationships can be perceived and learned. Because individuals have direct access to the information required to estimate distance and judge object locations, the distance estimates can be made by image scanning from the departure point to the end point. In contrast, when the space is learned by navigation, Thorndyke and Hayes-Roth assumed that the estimate requires a mental simulation of the route, and hence, the calculation of the angles and the length of each portion of the route. However, it has also been shown that greater familiarity with the surrounding space creates a qualitative change in the representation and a reorganization of the space, allowing for the transition from procedural to configurational knowledge (i.e., from knowledge for traversed routes to a more abstract, map-like representation). Using location estimates, Lloyd (1989) also compared the cognitive map of subjects who learned a space (the City of Columbia) by navigation with the cognitive map acquired by subjects through map learning (of an imaginary city with the same spatial structure as Columbia). The results differed from those obtained by Thorndyke and Hayes-Roth: errors were greater and the response times were longer for maps encoded through navigation than for maps encoded from cartographic maps. Lloyd interpreted these differences in terms of modes of storage and information processing assumed to depend upon the form in which spatial information is acquired. More specifically, Lloyd assumed that subjects who have learned a space by navigation have probably stored spatial information in a procedural form which is not transformed into a configurational form in spite of familiarity with the environment.

\section{Fluctuations}

Curiously, however, only one production per subject has been used in all of the experiments. This procedure is a natural one if the theoretical premise is that configurational representations are constructed and responses are obtained by scanning the image stored in memory. Indeed, there is no reason for a subject to give two different responses in this case. Thus, the distortion that consists of the systematic representation of a place where it is not located constitutes the best indicator of the state of the representation. In contrast, if a procedural form of information coding and retrieval is postulated, responses can intuitively be assumed to fluctuate and response times to vary. In other words, a subject in the same state processing under the same conditions will give different responses at different moments.

This idea of response fluctuation for a stable cognitive state (no learning or forgetting) is not new in psychology. In personality studies, for example, many researchers have questioned the consistently weak correlations found between an objective measure of behavior in one situation and the same measure taken either in another apparently strictly identical situation or assessed on a personality inventory. In this respect, Mischel (1968) states that behavioral consistency is not a proven reality, and that, on the contrary, the inconsistency regularly observed reflects a natural state (Mischel, 1969). In particular, although it is not exceptional to observe changes in individual characteristics across time, it is more difficult to treat changes across seemingly similar settings cross-sectionally. In other words, there is an illusion of immobility even though behavior is always changing. Relating this to our problem, the issue becomes whether or not the observed inaccuracy, usually called distortion, is partially due to response fluctuation. This question deserves attention because the use of a single response per subject naturally leads to emphasis upon interindividual, intersituation, or interstimulus differences, and obviously ignores response fluctuation.

This question has been investigated in both personality and neuroscience research, but it has been treated in a fundamentally different way. Epstein (1980), stressing that there are numerous sources of fluctuation and that the structure of those fluctuations is unknown, suggested avoiding the problem by increasing the number of responses per subject and averaging the data, as is conventionally done for subjects and stimuli. Epstein then added that this was apparently the only way not to unduly ascribe heuristic validity to a specific response. More recently, in the behavioral neurosciences, the study of fluctuations around stable states has provided information about the structure of these states, considered as attractors (Kay, Saltzman, \& Kelso, 1991; Schöner \& Kelso, 1988). Indeed, response variability may be an indication of the fact that certain dimensions are not taken into account, or that processing is purely stochastic or chaotic in nature (Pailhous \& Bonnard, 1992).

Thus, the study of response fluctuations may partially clarify the question of the equivalency of the cognitive processes involved in the two cases described above, that is, the reproduction from memory of the map of a fictitious space following the direct perception of the map (where all spatial relationships between objects are simultaneously present in the subject's visual field) and the reconstruction of the map of a real space learned by navigation (where the subject must organize a series of partial views and move from one frame of reference to another). Indeed, in the former case (direct perception of spatial relationships), if we assume that subjects reproduce the perceived configuration by scanning the memorized image, then response fluctuations can be expected to be relatively weak and homogeneous. In the latter case (reconstruction of the map following navigation), if we assume that familiarity with the environment initiates qualitative reorganization, then the fluctuations can be expected to be similar to those observed in direct map perception, and, again, to be equivalent for all locations. In contrast, if the information coding and/or retrieving processes are different-for example, procedural in the latter case-then the fluctuations can be expected to be greater and above all to vary by location.

In the present study, an experiment similar to the Thomdyke and Hayes-Roth (1982) and Lloyd (1989) experiments was conducted for the purpose of comparing differences 
not only in estimate accuracy but also in response fluctuation in each of the space-learning conditions. Among the diverse tasks available for the study of these questions, location estimation on a blank map appears to be one of the most direct methods of externalizing a spatial representation. This seems evident in the case of direct map perception, since the coding and response modes are strictly identical. In the case of a reconstruction, if repeated navigation within a space does indeed allow subjects to change from a route-map representation to a configurational or survey-map representation, then subjects who were born and have always lived in the same city should have no difficulty in using a survey map to perform the task.

\section{EXPERIMENT 1}

\section{Method}

Materials and Procedure. In six sessions, each separated by 3 days, subjects were presented individually with one of six different lists of 18 local landmarks situated in downtown Marseille. Each list was composed of 12 local landmarks which were considered as reference items (they remained the same in each trial) and 6 local landmarks which were different each time (filler items; see Figures 1A and 1B). This procedure (using filler items and spacing out the trials) was employed to avoid interference between the responses. The task proposed was to place these locations as accurately as possible on a sheet of paper $(36.5 \times 25.7 \mathrm{~cm})$ on which the limits of the downtown area (boulevards, coastline) had been repre-
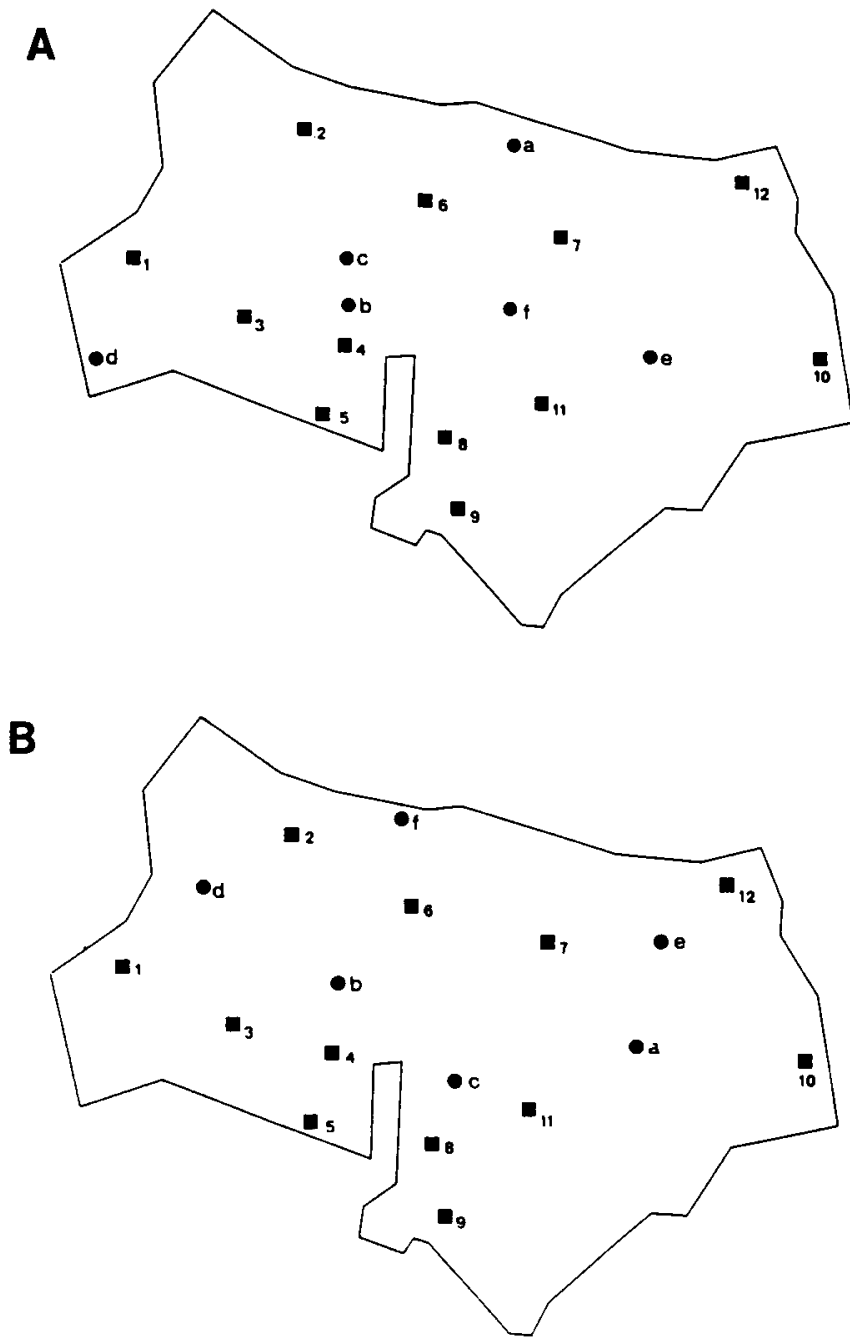

Figure 1. Two examples of the actual locations of different city sites. The enclosing solid lines represent the limits of the downtown area of Marseille. A and $B$ show the same reference locations but different filler locations. The reference ( $($ ) sites are: (1) Toursky Theater, (2) Palais Longchamp, (3) central fire station, (4) central Post Office, (5) La Major Cathedral, (6) central bus station, (7) business center, (8) St. Victor Abbey, (9) St. Georges Hall, (10) Palais de Chanot, (11) Notre Dame de la Garde, and (12) Congress Hall. The filler sites ( $\bullet$ ) are: A-(a) Conception Hospital, (b) Aix Square, (c) central railway station, (d) Cazemajou Square, (e) Insurance Building, and (f) Council Building. B - (a) Maison-Blanche Hospital, (b) Trade Unions Building, (c) Corderie Square, (d) Cadenat Square, (e) St. Joseph Hospital, and (f) Madeleine Hall. 
sented. No indication was given of the order in which the locations were to be placed, nor was there any time limit for the task. The subjects were allowed to change their responses as many times as they wished. They did not have access to a city map during the task and were specifically asked not to refer to a map for the duration of the experiment.

Because the task was repeated six times, six response sets were obtained for each subject. This allowed us to evaluate performance stability in terms of accuracy, and thereby to assess stability of behavior. The fluctuation of the responses was measured, considering each trial as a new source or reference (independently of the actual location) for comparing the other five trials. Six new values were then obtained to reflect response fluctuation. The computation of constant error and variable error (Schutz \& Roy, 1973) cannot be applied here. Constant-error computation requires signed estimates, which is meaningless in a two-dimensional situation. ${ }^{1}$

Subjects. Eleven subjects ( 6 women and 5 men) participated in the experiment. Their mean age was 39 years (range 31 to 46). All subjects had lived in the city (Marseille) since birth. At the time of the experiment, the subjects chosen had been in contact with each landmark several tens of times. They traveled downtown (on foot or by car) several times a week either for work or for recreation. Moreover, their knowledge of the different proposed sites was verified by asking them if they would be able to drive to each place. This suggested that, through repeated navigation, each subject had acquired a reasonable knowledge about the various locations and routes and that their representations would be stable (i.e., for the duration of the experiment, no learning or forgetting of spatial relationships were apt to occur). The subjects were volunteers and were not paid for their participation.

\section{Results}

The data were processed by two-dimensional regression analysis which computes a correlation coefficient, labeled $R 1$, between each layout produced by each subject and the real layout of the places.

There are various data-analysis programs which can perform these calculations (e.g., CONGRU developed by Oliver, 1970, and employed by Kosslyn et al., 1974, for example). The program used here (developed by Tobler, $1976,1977)$ was selected because it computes an indicator of mean distance (RMSE, or root mean square error) as well as indicators of individual distances for each site, and it extends the measures to the total area by interpolation. This provides a cartographic visualization of the deformations generated by estimate inaccuracy (see Appendix).

Analysis of congruity between real-map positions and subjects' estimates. The $R 1$ and $R M S E$ indicators were first computed for each subject and then averaged to give a mean value. Globally, for the six trials, the congruity level $(R 1)$ between the real-map locations and the subjects' estimates, as assessed by computing a correlation coefficient, reached a mean value of 0.81 with an $S D$ of 0.03 . The mean value of the RMSE indicator, which corresponds to the mean distortion (in distance) for all locations, was $2.11 \mathrm{~cm}$ (measured on the response sheet) with an $S D$ of $0.13 \mathrm{~cm}$. Given the scale of the map, this represents $457 \mathrm{~m}$ and an $S D$ of $28 \mathrm{~m}$.

A more detailed examination of the results showed a weak improvement in performance across trials (see Table 1). However, this improvement was not sufficient to reach the significance level $[F(5,50)=1.17$, n.s. $]$.
Table 1

Average Values of $R 1$ and $R M S E$ (in $M$ ) Indicators for the Six Successive Trials in Navigational Space

\begin{tabular}{lcccccc} 
& \multicolumn{7}{c}{ Trials } \\
\cline { 2 - 7 } & 1 & 2 & 3 & 4 & 5 & 6 \\
\hline$R 1$ & .76 & .80 & .80 & .82 & .82 & .83 \\
$R M S E$ & 509 & 464 & 464 & 442 & 444 & 425 \\
\hline
\end{tabular}

Note-The $R 1$ indicator is a correlation coefficient which gives the congruity level between real map positions and subjects' estimates. The $R M S E$ indicator gives the mean error in distance between real map positions and subjects' estimates.

The graphic representation of the estimate inaccuracy effect after interpolation showed that the area was deformed. While in some instances it appeared entirely "crumpled" (see Figure 2A), most of the time the deformations were confined to a smaller area with folds only in certain places (see Figure 2B).

Analysis of variance (subjects $\times$ locations) corroborated this finding by showing that the distance errors were greater for certain locations on all trials $[F(11,110)=$ $2.42, p<.01$ ] (see Table 2). This result reflects the heterogeneity of the subjects' knowledge of their surrounding space, which cannot be explained by familiarity with the various locations. Indeed, the high number of contacts with the landmarks makes an explanation linked to the frequencies of visiting unlikely.

Inaccuracy varied considerably from one location to the next, even within a given trial. Consequently, we needed to determine whether the distance error was of the same magnitude across locations and across trials. In other words, were the most inaccurately located places the same ones in other trials? The Kendall coefficient of concordance calculated for each subject showed that, on the average, the most poorly placed locations were the same across trials $(W=0.51)\left[\chi^{2}(11)=33.7, p<.001\right]$. Thus, the same pattern of errors was repeated from one trial to the next. Did this mean that the subjects were inaccurate but systematic in their errors?

Measurement of response fluctuation. The procedure used to measure response fluctuation consisted of taking one of the trials as a reference and the others as the image. By comparing the responses for a given subject independently of actual location, six new measures were obtained, with $R 1$ and $R M S E$ indicating response fluctuation. This procedure is much more precise than the determination of a confidence interval, which Gärling et al. (1981) used, because the confidence interval technique amounts to taking the subjects' responses at face value.

Globally, for all subjects and the six values of fluctuation, $R 1$ reached a mean value of 0.83 with an $S D$ of 0.03 . $R M S E$ had a mean value of $1.77 \mathrm{~cm}$ (383 $\mathrm{m}$ to scale), which corresponds to the average response fluctuation, with an $S D$ of $0.19 \mathrm{~cm}(41 \mathrm{~m}$ to scale).

A more detailed analysis of results showed a perceptible decrease in fluctuation (see Table 3). Here, contrary to what was observed for inaccuracy, this decrease was sufficient to reach the significance level $[F(5,50)=5.65, p<$ $.001]$. Thus, despite the precautions taken in the proce- 


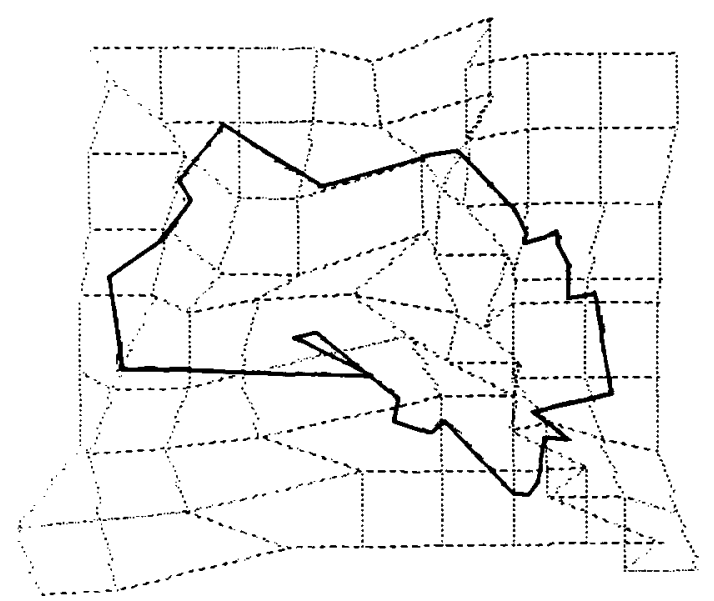

inaccuracy level? (2) Is there a link between the fluctuation in the subjects' estimated locations and the inaccuracy of those estimated locations? In other words, are the most fluctuating locations also the most inaccurate?

The answer to the first question is no. The results showed that response fluctuation and inaccuracy were similar in magnitude $(t=2.05$, n.s.) (see Table 5). The answer to the second question is also no. The most fluctuating locations were not always the most inaccurate. On the average, the correlation was not significant.

\section{Discussion}

Four important results were obtained from the present experiment. (1) Navigational space is not homogeneous,

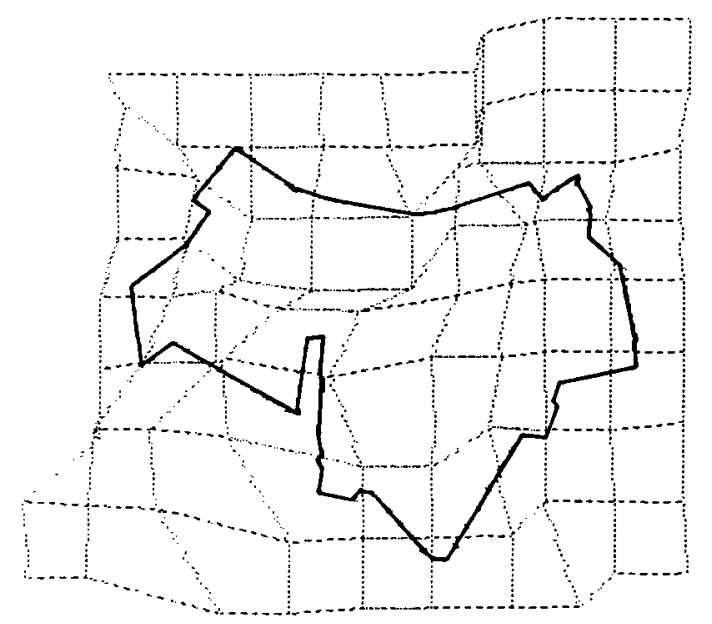

Table 2

Average Value of Inaccuracy (in Meters) for the Different Locations and the Six Trials (Navigational Space)

\begin{tabular}{|c|c|c|c|c|c|c|c|c|}
\hline \multirow[b]{2}{*}{ Locations } & \multicolumn{8}{|c|}{ Trials } \\
\hline & 1 & 2 & 3 & 4 & 5 & 6 & $M$ & $S D$ \\
\hline 1 & 394 & 390 & 396 & 394 & 429 & 375 & 397 & 18 \\
\hline 2 & 288 & 401 & 373 & 325 & 329 & 357 & 346 & 40 \\
\hline 3 & 396 & 414 & 483 & 509 & 390 & 344 & 423 & 62 \\
\hline 4 & 284 & 325 & 334 & 301 & 295 & 271 & 302 & 24 \\
\hline 5 & 336 & 299 & 275 & 238 & 234 & 227 & 268 & 43 \\
\hline 6 & 492 & 487 & 442 & 444 & 323 & 349 & 423 & 71 \\
\hline 7 & 340 & 262 & 388 & 362 & 373 & 412 & 356 & 52 \\
\hline 8 & 448 & 425 & 455 & 518 & 448 & 537 & 472 & 45 \\
\hline 9 & 684 & 578 & 565 & 442 & 490 & 442 & 534 & 94 \\
\hline 10 & 687 & 511 & 520 & 526 & 604 & 529 & 563 & 69 \\
\hline 11 & 453 & 349 & 340 & 264 & 264 & 290 & 327 & 72 \\
\hline 12 & 591 & 479 & 429 & 379 & 420 & 353 & 442 & 85 \\
\hline$M$ & 449 & 410 & 417 & 392 & 383 & 374 & & \\
\hline$S D$ & 141 & 93 & 83 & 98 & 104 & 94 & & \\
\hline
\end{tabular}

Table 3

Figure 2. Deformations produced by the inaccuracy of estimates in navigational space. Solid lines represent the limits of the downtown area.

dure (using filler items and spacing out the trials), an interference due to task repetition could not be completely avoided.

However, analysis of variance (subjects $\times$ locations) indicated that certain locations fluctuated more than others $[F(11,110)=1.96, p<.05]$ (see Table 4). In a space as complex as that of a city, subjects seem to respond inaccurately and their responses seem to fluctuate.

There was enough response fluctuation for the graphic representation after interpolation to reveal some degree of deformation, as it would if the map had been crumpled in some areas and enlarged in others (see Figure 3). One question raised by the significant variation in response fluctuation across locations and trials was whether or not the locations with the greatest fluctuations were the same from one trial to the next. Indeed, the Kendall coefficient of concordance showed that this effect was significant $(W=0.73)\left[\chi^{2}(11)=48.2, p<.001\right]$.

Two additional questions that arose were: (1) Does the response fluctuation level differ quantitatively from the

Average Values of $R 1$ and $R M S E$ (in Meters) Indicators for the Six Sources of Fluctuation in Navigational Space

\begin{tabular}{lcccccc}
\hline & \multicolumn{6}{c}{ Sources } \\
\cline { 2 - 7 } & 1 & 2 & 3 & 4 & 5 & 6 \\
\hline$R 1$ & .78 & .80 & .83 & .85 & .85 & .84 \\
$R M S E$ & 435 & 438 & 375 & 351 & 344 & 360 \\
\hline
\end{tabular}

Table 4

Average Value of Variability (in Meters) for the Different Locations and the Six Sources of Fluctuation (Navigational Space)

\begin{tabular}{|c|c|c|c|c|c|c|c|c|}
\hline \multirow[b]{2}{*}{ Locations } & \multicolumn{8}{|c|}{ Sources } \\
\hline & 1 & 2 & 3 & 4 & 5 & 6 & $M$ & $S D$ \\
\hline 1 & 328 & 323 & 289 & 299 & 290 & 297 & 304 & 17 \\
\hline 2 & 351 & 357 & 327 & 329 & 311 & 296 & 329 & 23 \\
\hline 3 & 378 & 365 & 333 & 309 & 274 & 268 & 321 & 46 \\
\hline 4 & 312 & 347 & 273 & 266 & 258 & 338 & 299 & 39 \\
\hline 5 & 261 & 228 & 212 & 211 & 197 & 193 & 217 & 25 \\
\hline 6 & 560 & 512 & 411 & 383 & 399 & 383 & 441 & 76 \\
\hline 7 & 409 & 422 & 322 & 346 & 300 & 343 & 357 & 48 \\
\hline 8 & 391 & 411 & 328 & 340 & 319 & 355 & 357 & 36 \\
\hline 9 & 390 & 423 & 412 & 351 & 321 & 354 & 375 & 40 \\
\hline 10 & 386 & 419 & 361 & 325 & 362 & 371 & 371 & 31 \\
\hline 11 & 413 & 363 & $\cdot 298$ & 289 & 286 & 284 & 322 & 54 \\
\hline 12 & 409 & 390 & 357 & 281 & 271 & 290 & 333 & 60 \\
\hline$M$ & 382 & 380 & 327 & 311 & 299 & 314 & & \\
\hline$S D$ & 72 & 69 & 56 & 46 & 51 & 54 & & \\
\hline
\end{tabular}




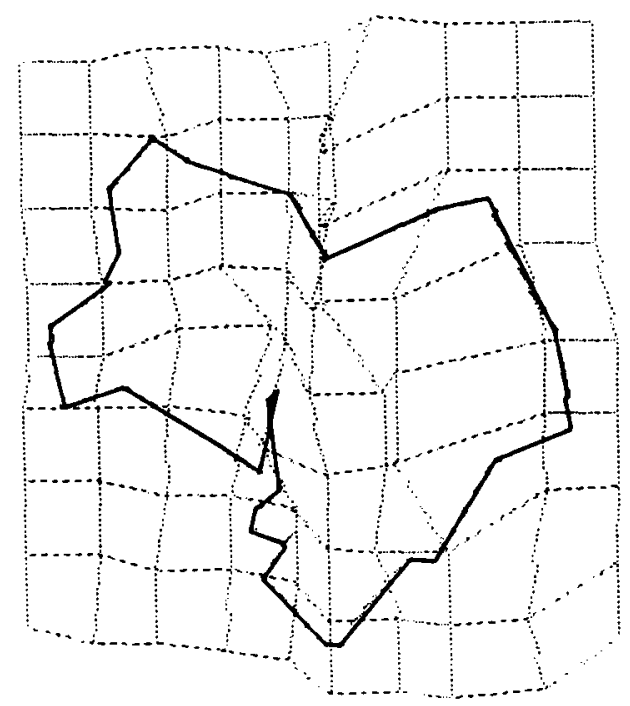

Figure 3. Deformations produced by response variability in navigational space. Solid lines represent the limits of the downtown area.

as can be seen from the variations in accuracy found from one location to the next. (2) In navigational spaces, subjects' estimates of locations vary, as shown by the response fluctuation observed across trials and the varying extent of that fluctuation, depending on the location. (3) Response fluctuation is of the same magnitude as inaccuracy. (4) Response fluctuation is not linked to inaccuracy.

Therefore, inaccuracy relative to real space cannot be considered as a good indicator of the cognitive mechanisms involved in spatial representation. Is response fluctuation an indication that a space was learned through navigation or, more globally, is it a general characteristic of spatial representation? A second experiment was conducted to try to answer this question.

\section{EXPERIMENT 2}

The first experiment provided insight into the role of response fluctuation in spatial information processing, allowing this fluctuation to be distinguished from inaccuracy or distortion. Using a strictly identical procedure with a space learned from a map, we approached the basic question of the structure of the cognitive processes involved in spatial information processing.

\section{Method}

Subjects. Two groups of subjects participated in this second experiment. The first experimental group contained the 11 subjects from the first experiment. The second, control group was composed of 8 new subjects ( 5 women and 3 men). Their mean age was 34 years (range 23 to 45 ). This second group was used to see whether the second experiment would be contaminated by the first.

Materials. The material was composed of three blank sheets of paper $(36.5 \times 25.7 \mathrm{~cm})$, each containing solid lines to represent the limits of three imaginary cities. Each city contained 12 locations, such as a church, a hospital, a supermarket, and so forth. The first imaginary city duplicated Marseille, except that the map was ro- tated 180 degrees and the names of the locations were changed (e.g., a cathedral in the real city became a supermarket in the imaginary city; see Figure 4A). The second and third imaginary cities had different boundaries, were presented in different orientations, and had different names for the locations (see Figures $4 B$ and $4 C$ ), but the spatial locations (i.e., the $x$ and $y$ coordinates) of the places shown on the three imaginary maps were strictly identical to the spatial locations of the 12 real places (reference items) in the first experiment. The use of three different imaginary cities was designed to avoid - or at least to minimize-the possibility that the subjects would learn the configuration and/or become saturated due to task repetition, thereby lowering performance. In other respects, the material used for all three imaginary cities and the real city was perfectly comparable with respect to the angles and distance ratios.

Procedure. The subjects were asked to study the map of the imaginary city for $3 \mathrm{~min}$. This amount of time is the minimal amount necessary to memorize 12 locations. The 3-min time period was established on the basis of a preliminary test using 4 different subjects and a map of the same size and with the same number of locations. The goal was not to ensure that subjects would be accurate, but rather that none of the locations would be forgotten. The subjects were asked to build an image of the map, that is, to prepare themselves to visualize the map with their eyes shut. After $3 \mathrm{~min}$, the map was removed and replaced by a response sheet (the same size as the map, but with only the boundaries drawn in). The subjects were then given as much time as they needed to reproduce the locations as precisely as possible using small pieces of cardboard $(1.2 \times 1.2 \mathrm{~cm})$. Each subject performed the task six times, twice for each imaginary city with a counterbalanced presentation. Every third day, in the same place and under the same conditions, the subjects again looked at one of the imaginary maps for $3 \mathrm{~min}$ and then placed the 12 locations as accurately as possible. Experiment 2 was performed about 1 week after the end of Experiment 1.

\section{Results and Discussion}

Analysis of the congruity level between the fictitious map and subjects' estimates. Globally, for the experimental group, the mean congruity level $(R 1)$ between the real positions and the subjects' estimates for the six trials was 0.92 with an $S D$ of 0.01 . The mean value of the RMSE indicator was $1.32 \mathrm{~cm}$ with an $S D$ of $0.08 \mathrm{~cm}$. For the control group, these values were $0.94(S D=0.01)$ and $1.09 \mathrm{~cm}(S D=0.09 \mathrm{~cm})$, respectively.

A more detailed analysis of results showed noteworthy stability of performance for both the experimental group and the control group. This stability was corroborated by analysis of variance $[F(5,50)=0.89$, n.s., for the first group and $F(5,35)=0.86$, n.s., for the second] (see Table 6). It must also be noted that the boundary and orientation changes had no effect on the subjects' performance

Table 5

Average Values of $R 1$ and $R M S E$ (in Meters) Indicators for Inaccuracy and Response Fluctuation in Navigational Space

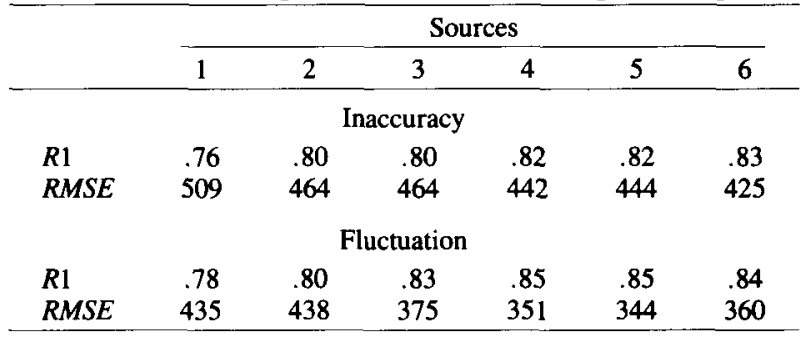




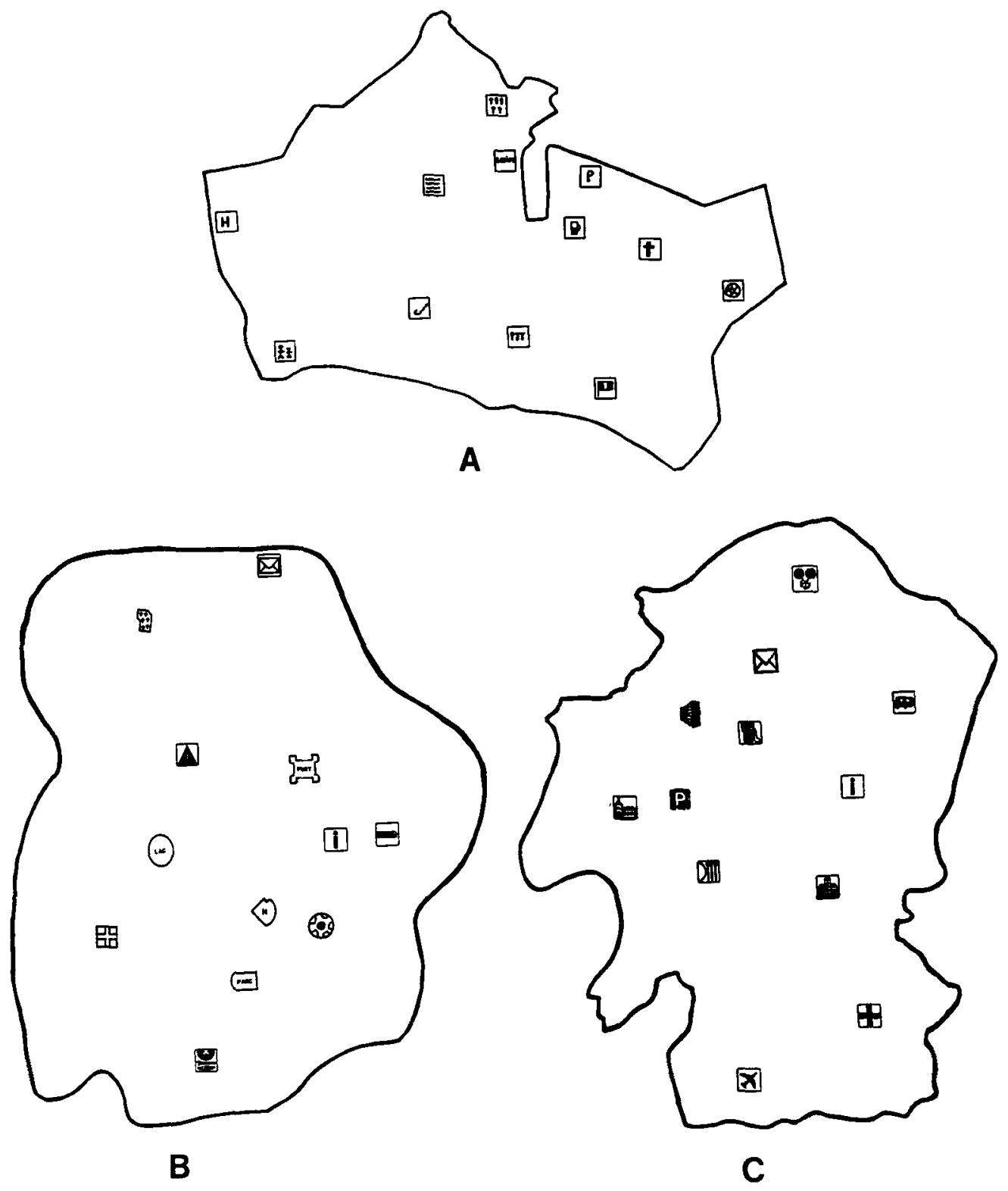

Figure 4. Maps of the three imaginary cities.

$[F(5,50)=0.66$, n.s., and $F(5,35)=2.32$, n.s. $]$. Thus, the difference in performance between the two groups was not significant $(t=1.97, \mathrm{n} . \mathrm{s}$.). The first experiment does not seem to have affected performance on the second experiment. In this respect, it is legitimate to analyze the performance of the experimental group in the second experiment, inasmuch as their performance was not tainted by an experimental bias.

Despite the lack of discrepancy between the perceived reality and the subjects' estimates, some deformations of the area appeared after interpolation. As can be seen in
Figure 5, the deformations were considerably smaller than in the first experiment and can be considered negligible.

Despite the proximity of the values, however, analysis of variance (subjects $\times$ locations) indicated, as it had in the first experiment, that error magnitude was greater for certain locations $[F(11,110)=2.42, p<.01]$.

Were the same locations placed inaccurately on different trials? The Kendall coefficient of concordance calculated for each subject showed that, on the average, in contrast to the former experiment, the most poorly placed locations on one trial were not the same on the other trials 
Table 6

Average Values of $R 1$ and $R M S E$ (in Centimeters) Indicators for the Six Successive Trials in Map-Learned Space

\begin{tabular}{|c|c|c|c|c|c|c|}
\hline & \multicolumn{6}{|c|}{ Trials } \\
\hline & 1 & 2 & 3 & 4 & 5 & 6 \\
\hline \multicolumn{7}{|c|}{ Experimental Subjects } \\
\hline $\begin{array}{l}R 1 \\
R M S E\end{array}$ & $\begin{array}{r}.93 \\
1.22\end{array}$ & $\begin{array}{r}.93 \\
1.27\end{array}$ & $\begin{array}{r}.92 \\
1.27\end{array}$ & $\begin{array}{r}.91 \\
1.42\end{array}$ & $\begin{array}{r}.92 \\
1.39\end{array}$ & $\begin{array}{r}.92 \\
1.33\end{array}$ \\
\hline \multicolumn{7}{|c|}{ Control Subjects } \\
\hline $\begin{array}{l}\boldsymbol{R} \mathbf{1} \\
\boldsymbol{R} M S E\end{array}$ & $\begin{array}{r}.94 \\
1.12\end{array}$ & $\begin{array}{r}.94 \\
1.17\end{array}$ & $\begin{array}{r}.93 \\
1.20\end{array}$ & $\begin{array}{r}.95 \\
1.04\end{array}$ & $\begin{array}{r}.95 \\
0.99\end{array}$ & $\begin{array}{r}.95 \\
1.00\end{array}$ \\
\hline
\end{tabular}

Table 7

Average Values of $R 1$ and $R M S E$ (in Centimeters) Indicators for the Six Sources of Fluctuation in Map-Learned Space

\begin{tabular}{lcccccc} 
& \multicolumn{7}{c}{ Sources } \\
& 1 & 2 & 3 & 4 & 5 & 6 \\
\hline$R 1$ & .89 & .88 & .87 & .87 & .86 & .87 \\
$R M S E$ & 1.53 & 1.57 & 1.68 & 1.71 & 1.80 & 1.68 \\
\hline
\end{tabular}

$(W=0.24)\left(\chi^{2}=15.84\right.$, n.s. $)$. Thus, subjects seemed to be clearly fluctuating in their responses. In what range were these fluctuations found?

Estimation of response fluctuation level. Globally, for the six values of fluctuation, $R 1$ reached a mean value of $0.87(S D=0.01)$. The mean value of the $R M S E$ indicator was $1.66 \mathrm{~cm}(S D=0.10 \mathrm{~cm})$.

A more detailed analysis of results showed that, despite the precautions taken in the procedure, there was a perceptible increase in fluctuation $[F(5,50)=3.09, p<.02]$ (see Table 7). The repetition of the task seemed to induce a weak saturation and hence reduced attention for the last trials.

On the graphic representation, the increase in response fluctuation was revealed by the greater degree of deformation of the surface, as can be seen in Figure 6 .

As in Experiment 1, was response fluctuation greater for some locations than for others? Analysis of variance (subjects $\times$ locations) indicated that, on the average, the response fluctuation level was equivalent across locations $[F(11,110)=1.53$, n.s. $]$. In other words, it tended to be the same for all locations.

Finally, response fluctuation was found to be greater than inaccuracy $[t(20)=2.9, p<.01]$, and the link between response fluctuation for a given location and the inaccuracy of the estimated placement of that location (relative to the real location) was found, on the average, to be significant $[R(10)=.62, p<.05]$.

Three important points stand out in the results of this second experiment:

1. The equivalent inaccuracy levels observed suggested that the performance of experimental and control subjects did not differ significantly. In this respect, the former experiment had no obvious influence on the latter.

2. In map-learned space, locations take variable positions, as shown by the response fluctuation observed across trials. However, the fluctuation magnitude was constant, regardless of location.

3. Mean response fluctuation was greater than inaccuracy, and fluctuation level increased with inaccuracy level. On the whole, there were few findings common to both experiments. Could it be that the underlying processes are not equivalent, despite the similarity of the spatial relationships?

The comparison of performance between navigational space and map-learned space in terms of inaccuracy and fluctuation showed that, on the average, subjects were both significantly more inaccurate $[t(20)=19.75, p<$ $.001]$ and more fluctuating $[t(20)=2.2, p<.05]$ in navigational space than in map-learned space. Furthermore, each location in the navigation-learned space directly corresponded to a location in the map-learned space, making a point-by-point comparison possible. The results showed that the locations placed with the greatest inaccuracy in the first experiment were not the same as the ones in the second experiment $(R=.27, \mathrm{n} . \mathrm{s}$. $)$. Likewise, the locations placed with the highest degree of fluctuation in the navigation-learned space were not those which fluctuated the most in the map-learned space $(R=.24$, n.s. $)$.

\section{GENERAL DISCUSSION}

These experiments were designed to answer two questions: (1) Can spatial representation be studied with only one production per subject? (2) Do the cognitive processes involved in spatial information processing differ according to the way in which the space is learned (Evans \& Pezdek, 1980; Lloyd, 1989)? Results of the two experiments showed that:

1. The fluctuations cannot be considered only as interindividual differences in estimates (Gale, 1980, 1983);

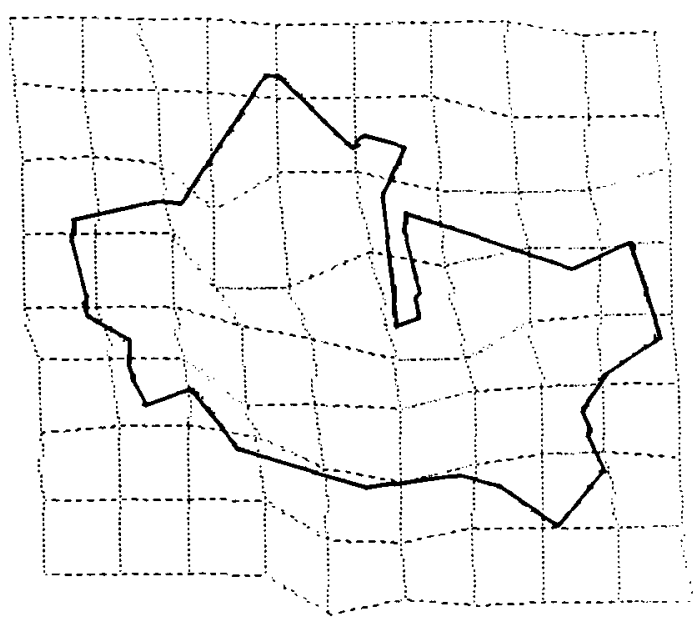

Figure 5. Deformations produced by the inaccuracy of estimates in map-learned space. The solid lines represent the limits of the imaginary city. 


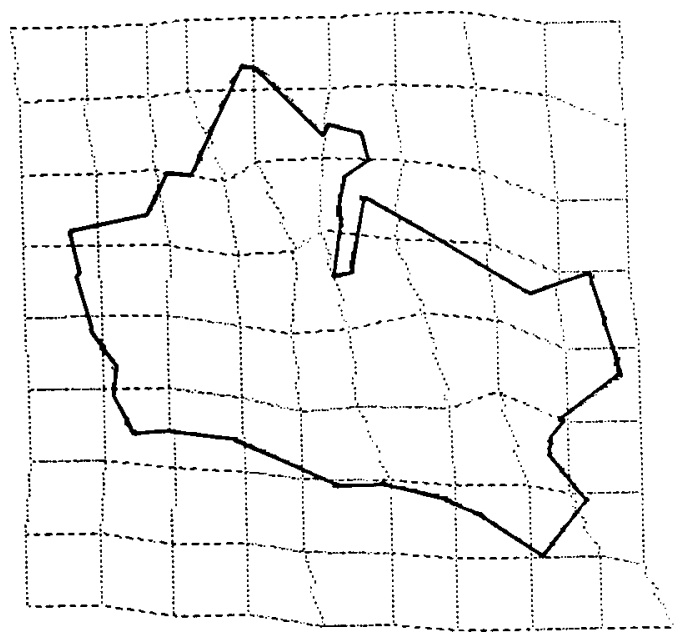

A

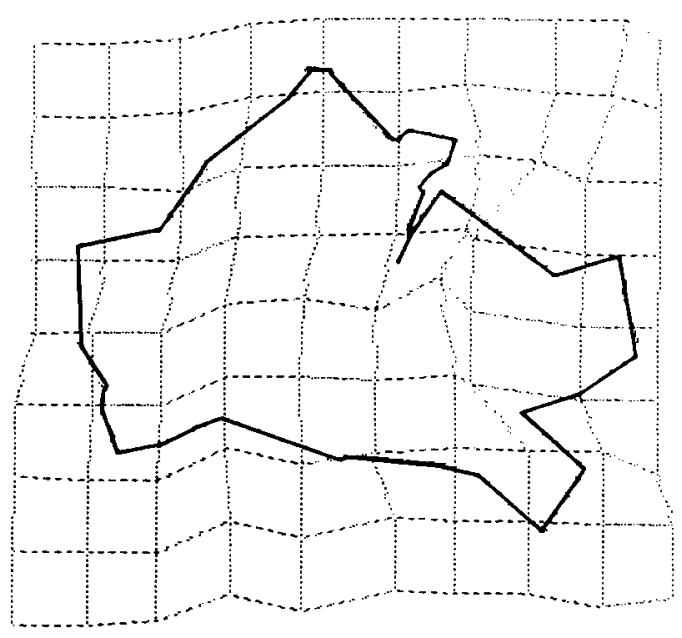

Figure 6. Deformations produced by response variability in maplearned space. (A) Trial 1 is taken as the source, and (B) Trial 6 is taken as the source. Solid lines represent the limits of the imaginary city.

they constitute a component of each subject's response and therefore a component of error. Consequently, spatial representation cannot be studied with only one production per subject because the measured discrepancy, generally assumed to reflect distortion, was nonsignificant.

2. The procedure proposed by Epstein (1980), which consists of multiplying and averaging the data to avoid assigning a heuristic value to a specific response, did not solve the problem posed here. Indeed, by averaging data, two parameters (distortion and fluctuation) that are a priori independent would have been mixed, even as, for their respective contributions to be evaluated, it is essential that they be distinguishable. With Epstein's procedure, a composite response would have been created which would have taken on a heuristic value that it does not deserve.

3. The repetition of trials showed that in navigational space the range of values for response inaccuracy, that is, distortion, is not different from (for example, larger than) the range of values for response fluctuation. On the average, the ratio of inaccuracy to fluctuation was 1.19. At first sight, what is usually called distortion could just as well be called fluctuation.

However, as can be seen in Figure 7A, for a subject close to the mean value of the group, a fluctuation response area (dotted line) is observed for each location. Two cases of distortion appeared for the locations situated on either side of the harbor (natural obstacle to navigation). When the real position of the location is included in the fluctuation area, which is the case for three of the places in Figure 7A, it is not possible to speak of distortion. Hence, these responses correspond to fluctuation. In this case, the distance between the real location of the place and the equilibrium position of the responses (solid line) does not constitute a case of distortion because it represents a valid estimate of the place. In contrast, for the two locations situated on either side of the harbor, the real position of the places is genuinely different from the equilibrium position of the responses, situated outside the fluctuation area. In this case, the distance between the real position and the equilibrium position (solid line) actually corresponds to a distortion, because the part of the line outside the fluctuation area does not fall in the range of potential estimates of the place.

Response fluctuation also affects spaces learned by map. The repetition of trials in the second experiment showed that the range of values was smaller for response inaccuracy than for response fluctuation. On the average, the inaccuracy-to-fluctuation ratio was 0.80 . It seems difficult to speak here about distortion, especially because response fluctuation was constant across locations. In Figure 7B, again for a subject close to the mean value of the group, it can be seen that when spatial relationships are simultaneously present in the visual field, the estimated locations are systematically placed around the real location, the latter always being included in the fluctuation area (i.e., lack of distortion). To this first difference between a space learned by navigation and a space learned by map, two others can be added.

4. In line with the hypothesis of a configurational representation which preserves the Euclidean properties of real space, Golledge (1987) suggested that distortion and fluctuation, two a priori independent dimensions, are in fact linked in the representation. According to Golledge, familiarity with the environment decreases the variability of the potential location of places, thus allowing for increasing metric precision. In other words, the more a subject knows a space, the greater estimated location accuracy and the lower the fluctuations. Inversely, the less familiar the subject is with the space, the more inaccurate and fluctuant the estimates will be. Our results invalidate this hypothesis for spaces learned by navigation, because the independence of these two dimensions was statistically confirmed. In contrast, our results confirmed this hypothesis for spaces learned by map. 

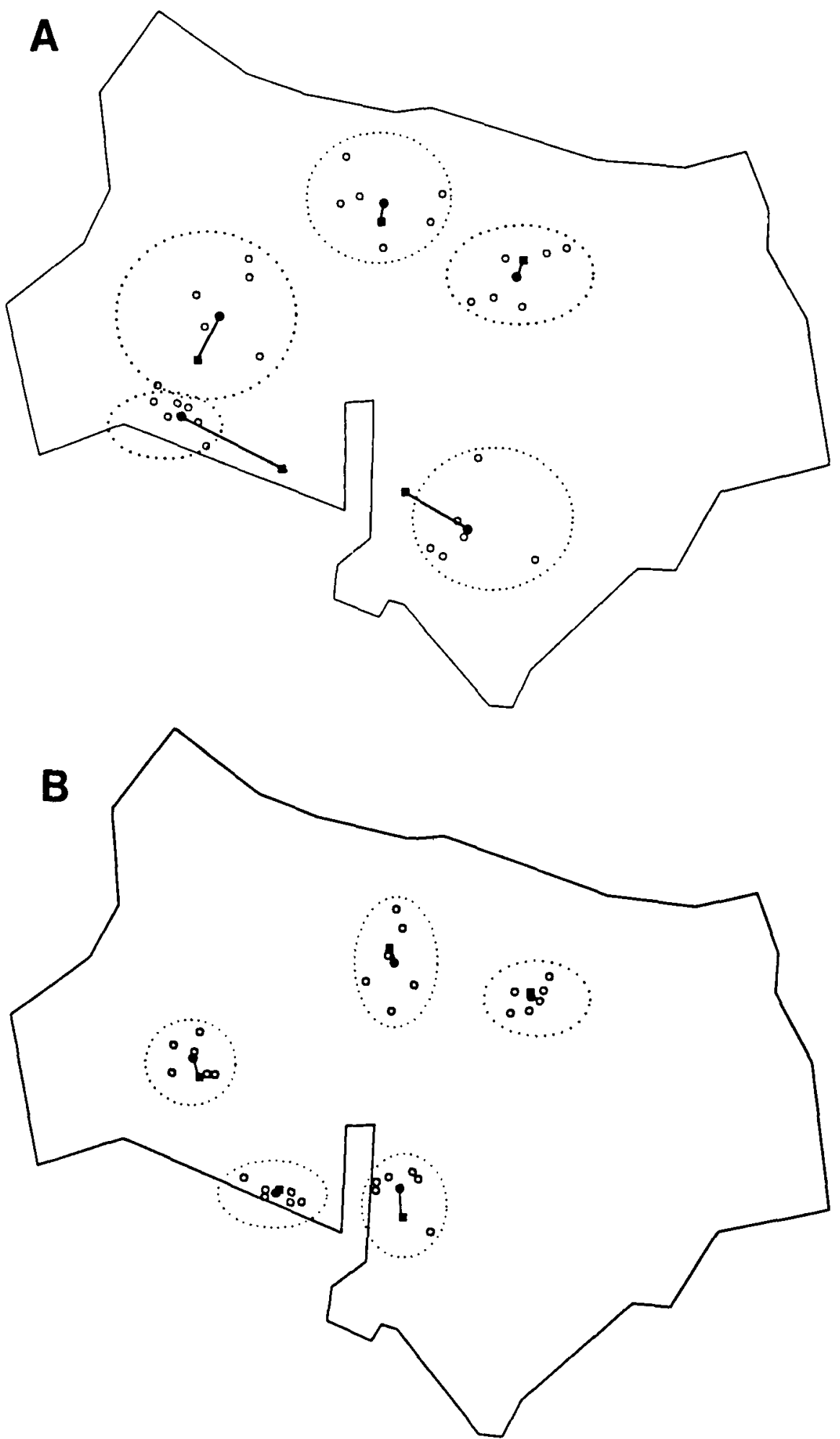

Figure 7. Spatial distribution of responses (A) in navigational space, and (B) in map-learned space. Filled squares represent actual locations, filled circles represent the equilibrium position of the estimated locations, open circles represent the estimated locations, dotted lines represent the fluctuation area, and solid lines, the distance between actual location and the equilibrium position of the location. 
5. Thorndyke and Hayes-Roth (1982) showed, for inaccuracy levels only, that while the differences between the two spaces are great and normal when the space learned by navigation is not very familiar, these differences decrease substantially after some months of intense navigation through the space. In this context, our results showing such dissimilar levels of inaccuracy and fluctuation for the two spaces with subjects who were born and had always lived in the same city are inexplicable. Although the subject's knowledge of small-scale spaces of low complexity, such as the ones used by Thorndyke and Hayes-Roth, can be qualitatively transformed from procedural knowledge to configurational knowledge (i.e, from knowledge for traversed routes to a more abstract maplike representation), making the environment translucent for them, this does not hold true for spaces as complex as those in a city. In this respect, our results tend to confirm those obtained by Lloyd (1989).

Finally, our results showed that response fluctuation depends on the location in navigational space, but is of constant magnitude in map-learned space. When subjects learn a space by map, the reproduction of the perceived configuration is done by visualizing and scanning the memorized mental image. Thus, it is difficult to understand why certain locations would fluctuate more than others. In contrast, for spaces learned by navigation, if the response is not based on, and subsequent to, the visualization of a previously organized image, differences in fluctuation from one location to another are perfectly imaginable.

Thus, the fluctuation of responses appears to be a fundamental element of spatial memory. Indeed, the presence of these fluctuations in two distinct tasks, as well as their importance (of the same or larger magnitude than inaccuracy), did not allow us to consider them as side effects of the system, or as some more or less perverse effects of the task. In biological systems, fluctuations are frequently of great functional importance. They not only provide evidence of the intrinsic flexibility of these systems (in our case, if a place has only an approximate position in the subject's mind, an obstacle on the way could be more easily avoided), but also show that this flexibility is a base for the plasticity of the system, that is, the architectural changes that can be observed in learning phases, for example. Learning leads more to a decrease in fluctuation-and thus, unfortunately, in flexibilitythan to a decrease in distortion. Therefore, as illustrated here by the places near the harbor, true functional distortions can be observed. What was shown here is that a distortion cannot be understood or observed without the adequate analysis of fluctuation for a stable state.

\section{REFERENCES}

Baird, J. C., Merrill, A. A., \& Tannenbaum, J. (1979). Studies of the cognitive representation of spatial relations: II. A familiar environment. Journal of Experimental Psychology: General, 108, 92-98.

BAIRD, J. C., WAGNER, M., \& Noma, E. (1982). Impossible cognitive spaces. Geographical Analysis, 14, 204-216.
BYRNE, R. W. (1979). Memory for urban geography. Quarterly Journal of Experimental Psychology, 31, 147-154.

CAUviN, C. (1984). Espaces cognitifs et transformations cartographiques: Les conditions de la comparaison des espaces cognitifs: de la carte aux configurations; exemples de l'espace urbain strasbourgeois. Thèse d'Etat es Lettres et Sciences Humaines, Strasbourg.

Courant, R. (1936). Differential and integral calculus (Vol. 2). New York: Wiley Interscience.

EPSTEIN, S. (1980). The stability of behavior: II. Implications for psychological research. American Psychologist, 35, 790-806.

Evans, G. W., \& Pezdek, K. (1980). Cognitive mapping: Knowledge of real-world distance and location information. Journal of Experimental Psychology: Human Learning \& Memory, 6, 13-24.

GALE, N. (1980). An analysis of the distortion and fuzziness of cognitive maps by location. Unpublished manuscript, University of California, Santa Barbara, Department of Geography.

GALE, N. (1983). Measuring cognitive maps: Methodological considerations from a cartographic perspective. In D. Amadeo, J. B. Griffin, \& J. J. Potter (Eds.), EDRA 1983. Proceedings of the Fourteenth International Conference of the Environmental Design Research Association (pp. 65-72). Lincoln: University of Nebraska.

GärLING, T., BööK, A., \& ERGEZEN, N. (1982). Memory for the spatial layout of the everyday physical environment: Differential rates of acquisition of different types of information. Scandinavian Journal of Psychology, 23, 23-35.

Gärling, T., BööK, A., Lindberg, E., \& Nilsson, T. (1981). Memory for the spatial layout of the everyday physical environment: Factors affecting rate of acquisition. Journal of Environmental Psychology, 1, 263-271.

GolleDGE, R. G. (1987). Environmental cognition. In D. Stokois \& I. Altman (Eds.), Handbook of environmental psychology (Vol. 1, pp. 131-174). New York: Wiley.

Golledge, R. G., Briggs, R., \& Demko, D. (1969). The configuration of distances in intraurban space. Proceedings of the Association of American Geographers, 1, 60-65.

GolledGe, R. G., \& HuberT, L. J. (1982). Some comments on nonEuclidean mental maps. Environment \& Planning, A, 14, 107-118.

Golledge, R. G., Smith, T. R., Pellegrino, J. W., Doherty, S., \& Marshall, S. P. (1985). A conceptual model and empirical analysis of children's acquisition of spatial knowledge. Journal of Environmental Psychology, 5, 125-152.

GOLLEDGE, R. G., \& SPECTOR, A. N. (1978). Comprehending the urban environment: Theory and practice. Geographical Analysis, 10, 403-426.

Kay, B. A., Saltzman, E. L., \& Kelso, J. A. S. (1991). Steady state and perturbed rhythmical movements: A dynamical analysis. Journal of Experimental Psychology: Human Perception \& Performance, 17, 183-197.

Kosslyn, S. M., Pick, H. L., \& Fariello, G. R. (1974). Cognitive maps in children and men. Child Development, 45, 707-716.

Kosslyn, S. M., \& Pomerantz, J. R. (1977). Imagery, propositions, and the form of internal representation. Cognitive Psychology, 9, 52-76.

KozlowsKI, L. T., \& BRYANT, K. J. (1977). Sense of direction, spatial orientation, and cognitive maps. Joumal of Experimental Psychology: Human Perception \& Performance, 4, 590-598.

LLOYD, R. (1989). Cognitive maps: Encoding and decoding information. Annals of the Association of American Geographers, 79, 101-124.

MisCHEL, W. (1968). Personality and assessment. New York: Wiley.

MischeL, W. (1969). Continuity and change in personality. American Psychologist, 24, 1012-1018.

MOAR, I., \& BOWER, G. H. (1983). Inconsistency in spatial knowledge. Memory \& Cognition, 11, 107-113.

OLIVER, D. (1970). Metrics for comparison of multidimensional scalings. Unpublished manuscript, Harvard University.

Pailhous, J., \& BonNard, M. (1992). Steady-state variability of human gait: Role of optical flow. Behavioural Brain Research, 47, 181-190.

Richarpson, G. D. (1981). The appropriateness of using various Minkowskian metrics for representing cognitive configuration. Environment \& Planning, $A, 13,475-485$. 
SCHÖNER, G., \& KELso, J. A. S. (1988). A dynamic pattern theory of behavioral change. Journal of Theoretical Biology, 135, 501-524.

SchuTz, R. W., Roy, E. R. (1973). Absolute error: The devil in disguise. Joumal of Motor Behavior, 5, 141-153.

ThorNdyke, P. W., \& HAYes-Roth, B. (1982). Differences in spatial knowledge acquired from maps and navigation. Cognitive Psychology, 14, 560-589.

TOBLER, W. R. (1965). Computation of the correspondence of geographical patterns. Papers of the Regional Science Association, 15, 131-139.

TobleR, W. R. (1976). The geometry of mental maps. In G. Rushton \& R. G. Golledge (Eds.), Spatial choice and spatial behavior (pp. 6982). Columbus: Ohio State University Press.

TOBLER, W. R. (1977). Bidimensional regression: A computer program. Unpublished manuscript, Unjversity of California, Santa Barbara.

TOBLER, W. R. (1978a). Comparing figures by regression. Paper presented at the ACM siggraph meeting, Atlanta.

TOBLER, W. R. (1978b). Comparison of plane forms. Geographical Analysis, 2, 154-162.

\section{NOTE}

1. Constant-error and variable-error computation required, in our case (two-dimensional situation), the separation of the $x$ - and $y$-axes. This could be of interest if one assumed that the space was not isotropic and if one were interested in the study of distortions and/or fluctuations on the dominant axis. That was not our purpose. Moreover, separating the two dimensions deleted essential information about the distance error.

\section{APPENDIX}

(This document is a kindly authorized reproduction of the work done by Colette Cauvin, 1984.)

The problem to be solved here is how to compare two surfaces or two irregular spatial configurations on which points are identified and labeled by coordinates in a given coordinate system (Cartesian, polar, etc.). In the case that interests us here, a geographic map with a predefined scale and orientation on which points are labeled with $x$ and $y$ coordinates is compared with subject-generated configurations involving the same points placed at variable locations, with irregular variations in scale and orientation.

Two-dimensional regression, a procedure designed by Tobler $(1965,1977,1978 \mathrm{a}, 1978 \mathrm{~b})$ to solve this problem, can be used to compare homologous configurations of points. It involves two fundamental steps and requires the calculation of numerous indices and an elaborate system of maps. For the sake of clarity, the basic principle behind this method will be presented first. Then a brief description of the steps will be given.

\section{BASIC PRINCIPLE}

The method presented here is based on the following principle:

Let $Z$ and $W$ be two spatial configurations, where $Z$ is the original configuration containing $n$ points with coordinates $\left(X_{i}, Y_{i}\right)$ (here, the geographic map) and $W$ is the "image" configuration with $n$ homologous points with coordinates $\left(U_{i}, V_{i}\right)$ (here, the subject's configuration).

Each point is thus defined by two numbers on each of the surfaces. Its displacement between the two surfaces can be schematically represented by a vector $\left(X_{i}, Y_{i}\right),\left(U_{i}, V_{i}\right)$ (see Figure Al).

The problem is to find the relationship between the two sets, $Z$ and $W$, that is, the function $\widehat{W}$ of $Z$, which is the best fit for the observed set $W$. The two surfaces are fit to each other as variables are fit in unidimensional regression. The best fit is found by minimizing the differences between the observed surface $W$ and the fitted surface $\widehat{W}$.

But this method is not limited to finding the best fit for the points selected on the surfaces. It includes a second step, which extends the results obtained for the selected points to the entire surface under study. Accordingly, deformations can be located and measured at all points on the surface, not just at a few specific points.

This method yields four distinct homologous surfaces: the original surface, $Z$ (the reference surface); the initial observed image, $W$; the fitted image, $\widehat{W}$, where selected points can be compared; and the interpolated image, $\widehat{\widehat{W}}$, where the entire surface can be studied.

\section{STEPS}

\section{The Fitting Principle}

Finding the best fit consists of relating $W$ to $Z$ by a function $\widehat{W}=f(Z)$, such that the projection of $Z$ onto $\widehat{W}$ is as close as possible to the projection of $Z$ onto $W$. As close as possible is interpreted in terms of least squares. In other words, the following quantity must be minimized:

$$
1 / n \sum_{k=1}^{n}(W-\hat{W})^{2}=1 / n \sum_{k=1}^{n}(W-f(Z))^{2},
$$

where $K$ varies from 1 to $n$.

The goodness of fit is measured as in one-dimensional regression by a ratio of the following type:

$$
1-\frac{\sum_{k=1}^{n}(W k-\hat{W} k)^{2}}{\sum_{k=1}^{n}(W k-\bar{W} k)^{2}}=1-\frac{\text { explained variance }}{\text { total variance }} .
$$

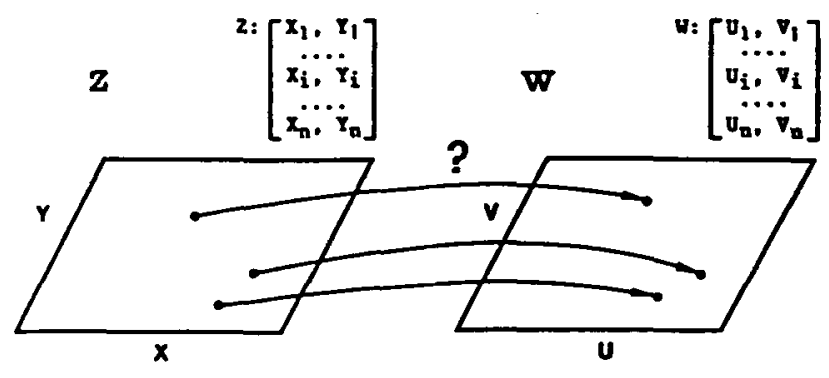

Figure A1. Two-dimensional regression: the problem to be solved. 
The fit is linear, which means that straight lines on the original surface are straight lines on the image surface. The best fit function is the projection of $Z$ onto $W$ such that the following statements are equivalent:

$$
\left(\begin{array}{l}
X \\
Y
\end{array}\right) \rightarrow\left(\begin{array}{l}
U \\
V
\end{array}\right) \quad\left\{\begin{array}{l}
U=f(X, Y) \\
V=g(X, Y)
\end{array}\right.
$$

The linear transformation used is a euclidian transformation described by the following system of equations, expressed in two equivalent manners:

$$
\left(\begin{array}{l}
U \\
V
\end{array}\right)=\left(\begin{array}{rr}
a_{11} & -a_{12} \\
a_{21} & a_{22}
\end{array}\right)\left(\begin{array}{l}
X \\
Y
\end{array}\right)+\left(\begin{array}{l}
b_{1} \\
b_{2}
\end{array}\right) \begin{cases}U & a_{11} X-a_{12} Y+b_{1} \\
V & a_{21} X+a_{22} Y+b_{2}\end{cases}
$$

Only four parameters need be defined, due to the symmetry of the coefficients of the independent variables (here, $X$ and $Y$ ):

$$
a_{11}=a_{22} \text { and } a_{12}=-a_{21} \text {. }
$$

The deformations are uniform, that is, identical in all directions. Thus, the "slope" of the regression can be calculated using the following formula:

$$
\left(a_{11}^{2}+a_{12}^{2}\right)^{1 / 2}
$$

This slope, in fact, represents a scale because it indicates the expected change in $W$. It reflects the enlargement or reduction ratio between the two surfaces when their origins and orientations coincide. Angles are not changed by the transformations; only lengths and areas are modified (i.e., multiplied by a constant).

\section{The Fitting Procedure}

The fitting procedure involves three operations which determine the transformation that makes the two surfaces coincide the "best": a translation, a rotation, a scale change, or a homothetic modification.

The least squares solution amounts to "determining the coefficients $\mathrm{A}$ and $\mathrm{B}$ of the transformation $\hat{W}=\mathrm{AZ}+\mathrm{B}$ such that the residues are minimized' (Courant, 1936). Thus, coefficients $a$ and $b(a 11, a 12$, and $b 1, b 2)$ must be found in order to minimize the above quantity:

$$
1 / n \sum_{k=1}^{n}(W-\hat{W})^{2}=1 / n \sum_{k=1}^{n}(W-f(Z))^{2} .
$$

The solution requires the use of a matrix where the system of equations looks as follows for a univariate bidimensional regression:

$$
\left[\begin{array}{cccc}
n & 0 & \Sigma X & \Sigma Y \\
0 & n & \Sigma Y & \Sigma X \\
\Sigma X & \Sigma Y & \Sigma(X+Y) & 0 \\
\Sigma Y & -\Sigma X & 0 & \Sigma(X+Y)
\end{array}\right]\left[\begin{array}{l}
b_{1} \\
b_{2} \\
a_{1} \\
a_{2}
\end{array}\right]\left[\begin{array}{c}
\Sigma U \\
\Sigma V \\
\Sigma(U X+V Y) \\
\Sigma(U Y+V X)
\end{array}\right]
$$

\section{The Interpolation}

The purpose of this step is to extend the measures to the entire surface under study in order to obtain deformation indices for all points therein. It also generates a distortion map.

Tobler $(1977,1978 \mathrm{a}, 1978 \mathrm{~b})$ has discussed the choice of the interpolation procedure. In general, however, the procedure consists of interpolating the known values of certain points on the surface, to obtain values for all nodes on the interpolation grid superimposed over the surface. This step involves essentially two operations: (1) determine the characteristics of the interpolation grid, and (2) calculate the new values assigned to all nodes on the grid.

Determining the interpolation grid. Four operations are necessary: (1) Calculate the area of the grid by taking the product of the maximum coordinate range on each axis. The grid should go beyond the surface studied. The values are usually increased by $5 \%$. (2) Determine the criteria for defining the grid (maximum size of the mesh). (3) Calculate the size of the mesh. (4) Calculate the number of rows and columns in the grid.

Calculating the new values. The vectors that express the discrepancy between the source points and the target points define a field of force in which a vector is applied to each node in the interpolation grid. In this case, each node is subjected to a force which is a combination of all the applicable individual forces, and whose norm is inversely proportional to the distance from that node.
(Manuscript received May 2, 1992; revision accepted for publication May 12, 1993.) 